Original Research Paper

\title{
Image Binarization Using Block Boundary Pixels Mean
}

\author{
O. Imocha Singh and T. Romen Singh \\ Department of Computer Sciences, Manipur University, Imphal, Manipur, India
}

Corresponding Author:

O. Imocha Singh

Department of Computer

Science, Manipur University,

Imphal, Manipur, India

Email: imocha2007@rediffmal.com

\begin{abstract}
Input image pixels classification into two intensities like black and white as foreground and background respectively refers to image binarization. Binarization with a local threshold value is termed as adaptive image binarization. Locally adaptive thresholding techniques are local pixels dependent process. Local environment dependent process is normally time consuming one and hence its computational time complexity is also local region dependent. Adaptation depends on the local region contrast condition. Low contrast region may not be adapted correctly. This article presents a new locally adaptive fast binarization technique applied on contrast stretched domain of an input image. The local adaptation is based on the mean of the 9 local block boundary pixels. As it associates only 9 pixels for mean calculation, its computational time complexity is free from local reason block size. As it applies on contrast stretched domain image, it can adapt low contrast region for binarization while other techniques fail. Not only its low contrast adapting capability, it has a local block size free computational time complexity. Hence it is more convenient to adapt the low contrast region very fast as compared with other techniques. From the experimental results, it is observed that it yields fast better result than other related local techniques.
\end{abstract}

Keywords: Image Binarization, Block Boundary Mean, Adaptive

\section{Introduction}

Input image pixels classification into two intensities like black and white is termed as binarization and it is very important in image processing specially in document image processing. The binarization which depends on the local region neighbouring pixels is called local adaptive binarization technique. In local techniques a certain threshold value is associated for each pixel to separate them into the two classes. Effective binarization process requires a suitable threshold value. Suitable threshold value can be searched in different ways and the ways can be broadly categorised as global, local and hybrid binarization.

The whole image is binarized using a single threshold value in global techniques (Otsu, 1979; White and Rohrer, 1983; Chi et al., 1996; Cattoni et al., 1998; Viola and Jones, 2004; Shafait et al., 2006). Grey value of each pixel is compared with the single threshold value for binarization. For typical scanned document images, it is very fast and gives good result as it compare with a single threshold value. Global techniques suffer from marginal noise along the page borders of inconsistent illumination documents images. Local techniques can solve this problem.
In local techniques, different threshold values are estimated for each pixel depending on the grey values of the neighbouring pixels. Since the threshold value estimation is based on neighbouring pixels, computational time consumption depends on the local block size. The techniques of (Chow and Kaneko, 1972; Bernsen, 1986; Niblack, 1986; Mardia and Hainsworth, 1988; Taxt et al., 1989; Yanowitz and Bruckstein, 1989; Eikvil et al., 1991; Sauvola and Pietikainen, 2000; O.I. Singh et al., 2012) belong to this category. Some techniques use integral sum image generation as prior process so as to calculate local mean easily. T.R. Singh et al. $(2011 ; 2012)$ use integral sum image to calculate local mean and hence their computational time consumption is free from local block size. So these techniques are comparatively faster than others while the binarized results are almost similar.

In the hybrid techniques (O'Gorman, 1994; Liu and Li, 1997), both the global and local thresholds are considered as combine information for the binarization. This technique is suitable for inconsistent illuminated image.

This paper describes a fast locally adaptive binarization algorithm which is applied on contrast stretched domain image of an input image with a new local mean technique which has fixed computational 
time. The result is compared with other techniques and found outperforms regarding binarized result as well as computational time factor.

\section{Related Works}

\section{Local Mean Calculation}

Most of the local adaptive techniques associate local mean of pixels within a $w \times w$ local block. Local mean calculation is time consuming process. So as to reduce time consumption, many techniques associate integral sum calculation as prior processes.

\section{Local Arithmetic Mean}

The local arithmetic mean (LAM) of pixels within a $w \times w$ odd order block can be calculated within an image I as follows:

$$
m(x, y)=\frac{1}{w^{2}} \sum_{i=x-c}^{x+c} \sum_{j=y-c}^{y+c} I(i, j)
$$

Where:

$$
c=\frac{w-1}{2}(\mathrm{x}, \mathrm{y})
$$

$(\mathrm{x}, \mathrm{y})$ is the coordinate of the central pixel of the block within the image $I$.

Since the calculation involves all the pixels within the block, its computational time depends on the block size $w$. Hence any local techniques (Chow and Kaneko, 1972; Bernsen, 1986; Niblack, 1986; Eikvil et al., 1991; Sauvola and Pietikainen, 2000; O.I. Singh et al., 2012) which associates LAM, has a local block size dependent computational time. So as to free from block size $w$, some techniques (T.R. Singh et al., 2011; T.R. Singh et al., 2012) used integral sum or average image as prior process to calculate $L A M$ easily.

\section{Local Binarization Techniques}

In local binarization techniques, each pixel is transformed either in background or foreground by comparing with a local threshold $T(x, y)$ as:

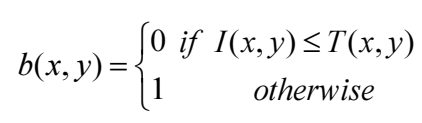

Where:

$$
\begin{aligned}
b(x, y) \in\{0,1\}= & \text { The binarized image } \\
I(x, y) \in[0,1]= & \text { The intensity of a pixel at location }(x, y) \\
& \text { of the input image } I
\end{aligned}
$$

Local threshold $T(x, y)$ is computed for each pixel based on some local statistics such as mean, variance, or standard deviation as discussed in the next subsection. Local statistical functions are applied only on the neighboring pixels within a $w \times w$ local window and hence its computational time complexity is $O\left(w^{2} \times n^{2}\right)$ for an image $I_{n \times n}$.

\section{Local Variance Methods}

Niblack (1986; Sauvola and Pietikainen, 2000; T.R. Singh et al., 2011; 2012) use the local variance technique. In these methods, the first two associate local mean $m(x, y)$ and standard deviation $\delta(x, y)$ while the last two associate only local mean $m(x, y)$ within a $w \times w$ window.

\section{Niblack's Technique}

In this method the local threshold value $T(x, y)$ at $(x, y)$ is calculated as:

$T(x, y)=m(x, y)+k \delta(x, y)$

Where:

$m(x, y)$ and $\delta(x, y) \quad=$ The local mean and standard deviation of the pixels inside the local window

$k$

$=\mathrm{A}$ bias

The result is satisfactory at $k=-0.2$ and $w=15$. The local mean $m(x, y)$ and standard deviation $\delta(x, y)$ adapt the threshold value based on the contrast in the local neighboring pixels. The bias $k$ controls the adaptation level varying the threshold value as in Fig. 1.

\section{Sauvola's Technique}

In Sauvola and Pietikainen's, 2000 method the threshold is calculated using the mean $m(x, y)$ and standard deviation and $\delta(x, y)$ of the pixels within a $w \times w$ window as:

$$
T(x, y)=m(x, y)\left[1+k\left(\frac{\delta(x, y)}{R}-1\right)\right]
$$

Where:

$R \quad=$ The maximum value of the standard deviation $(R=128$ for a grayscale document)

$k \in[0.01,0.5]=\mathrm{A}$ bias which control background noise as in Fig. 1

\section{Local Gray Range Method}

Bernsen (1986; O.I. Singh et al., 2012) use the local gray range technique. In these techniques the range between the maximum and minimum pixel gray range within the local window is used to determine the threshold value. 


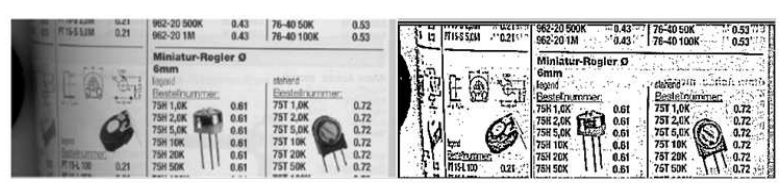

a. Original

b. $k=0.02$

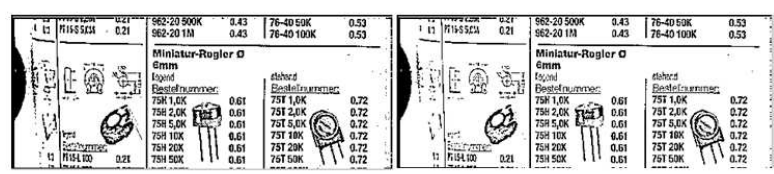

c. $k=0.06$

d. $k=0.1$

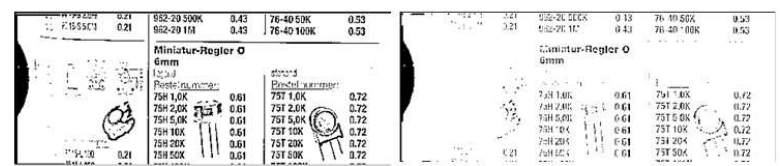

e. $k=0.2$

f. $k=0.4$

Fig. 1: Different levels control of background and foreground at different values of $k$

\section{Bernsen's Technique}

In this method the local threshold value $T(x, y)$ at $(x, y)$ is calculated within a local $w \times w$ window as:

$$
T(x, y)=\left\{\begin{array}{ccc}
0.5\left(I_{\max }+I_{\min }\right) & \text { if } & C \geq 15 \\
G & \text { otherwise }
\end{array}\right.
$$

Where:

$I_{\max }$ and $I_{\min }=$ Represent the maximum and minimum grey values within the local window

C

$=I_{\max }-I_{\min }$

$G$

$=$ Global threshold value

This method is also dealing with local window and hence its computation time is also local window size $w$ dependent like others and found satisfactory at $w=31$.

\section{O.I. Singh's Technique}

O.I. Singh et al. (2012) proposed a new technique of binarization which uses local contrast and mean to determine the local threshold and expressed as:

$T(x, y)=k\left[m(x, y)+\left(I_{\max }-I_{\min }\right)(1-I(x, y)]\right.$

Where:

$k \in(0,1)=$ background control constant.

$m(x, y) \quad=$ local mean.

$I_{\max }$ and $I_{\min }=$ the local maximum and minimum pixel values within a $w \times w$ local window.

$\mathrm{I}(x, y)=$ the concerned pixel value.

\section{Our Technique}

Our technique (OT) presents a new adaptive image binarization technique which is applied on contrast stretched domain with a 9 pixels only new local block boundary pixels mean.

\begin{tabular}{|c|c|c|}
\hline$(\mathrm{i}-1, \mathrm{j}-1)$ & $(\mathrm{i}-1, \mathrm{j})$ & $(\mathrm{i}-1, \mathrm{j}+1)$ \\
\hline$(\mathrm{i}, \mathrm{j}-1)$ & $(\mathrm{i}, \mathrm{j})$ & $(\mathrm{i}, \mathrm{j}+1)$ \\
\hline$(\mathrm{i}+1, \mathrm{j}-1)$ & $(\mathrm{i}+1, \mathrm{j})$ & $(\mathrm{i}+1, \mathrm{j}+1)$ \\
\hline
\end{tabular}

a. Block of size $w=3$

\begin{tabular}{|c|l|l|l|l|l|l|}
\hline$(i-3, j-3)$ & & & $(i-3, j)$ & & & $(i-3, j+3)$ \\
\hline & & & & & & \\
\hline$(i, j-3)$ & & & $(i, j)$ & & & $(i, j+3)$ \\
\hline & & & & & & \\
\hline & & & & & & \\
\hline$(i+3, j-3)$ & & & $(i+3, j)$ & & & $(i+3, j+3)$ \\
\hline
\end{tabular}

b. Block of size $w=7$

Fig. 2: Block boundary pixels at different block size $w$

\section{Block Boundary Pixels Mean}

Block boundary pixels mean(BBPM) is the mean of 9 pixels within a local block $\mathrm{B}_{w \times w}$. It will represent the mean of all pixels within the block without regarding the block size $w$ of odd order. It associates only the central pixel and another 8 boundary pixels as shown in Fig. 2 . Hence its computational time is fixed to the 9 values calculation and free from the block size $w$.

BBPM $m_{b}(x, y)$ of a local block of size $\mathrm{w}$ within an image I can be calculated as follows:

$$
\begin{aligned}
& m_{b}(x, y)=\frac{1}{9}[I(x-c, y-c)+I(x-c, y+c) \\
& +I(x+c, y-c)+I(x+c, y+c)+I(x, y) \\
& +I(x-c, y)+I(x, y-c)+I(x, y+c) \\
& +I(x+c, y)
\end{aligned}
$$

Where:

$$
c=\frac{w-1}{2}(\mathrm{x}, \mathrm{y})
$$

$(x, y)$ is the coordinate of the central pixel of the block within the image $I$.

BBPM is different from LAM and hence threshold values determined by using LAM and BBPM are different. Figure 3 shows the different threshold values determined by Sauvola and OT at different windows size. If $w=3$, there is no difference between the techniques. Even the threshold values are different it doesn't effect to the binarized result considerably. 


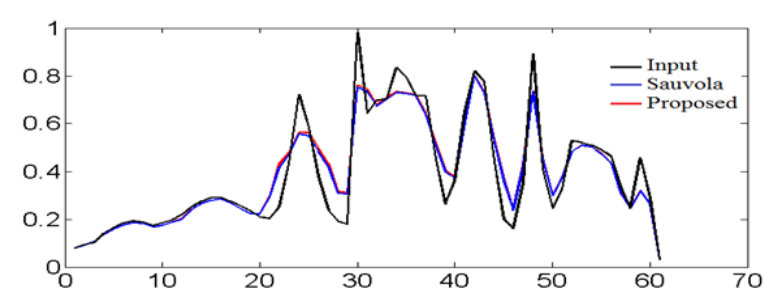

a. $A$ Structures of thresholds at $w=3$
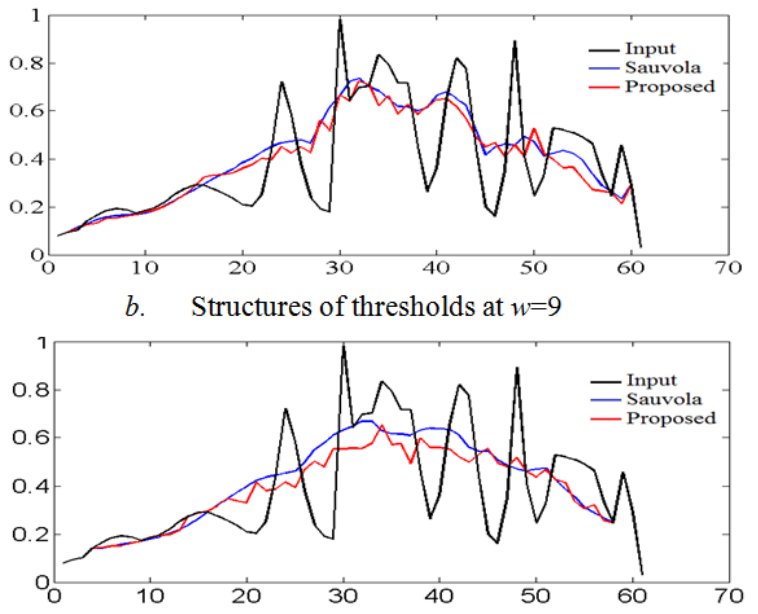

c. Structures of thresholds at $w=15$

Fig. 3: Different structures of thresholds by Sauvola with local mean and proposed technique with BBPM. at different local block size

\section{Contrast Stretching}

In low contrast images, it is failed to distinguish between the foreground and background pixels for binarization. This case is very common in document mages binarization. So as to remove the background noise as well as the foreground becoming more prominent, contrast stretching is applied on the input image $I$ as prior process. It makes more convenient to distinguish between background and foreground pixels while binarisation using a threshold value. Contrast stretching is carried out as:

$$
S(x, y)=\frac{I(x, y)^{2}\left(k_{s}+1\right)}{k_{s}}
$$

where $\{S, I\} \in[0,1]$ represents the stretched and input image respectively while $k_{s}>0$ is a constant which can control the level of stretching.

Figure 4 shows the different controls at different values of $k_{s}$ The lower the value of $k_{s}$ will give the more stretching between background and foreground.

\section{Thresholding and Binarization}

Once the contrast stretched domain image $S$ is determined, a new thresholding process is applied on $S$ for binarization. The threshold value $T(x, y)$ for binarization is calculated for each pixel as:

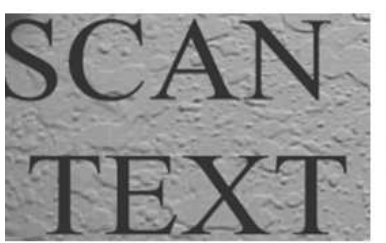

a. Original

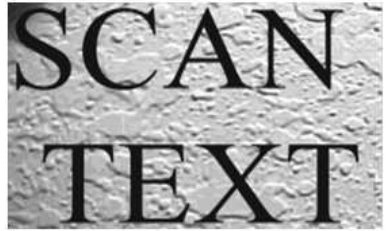

c. $k_{s}=1$

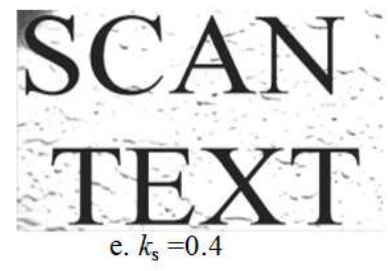

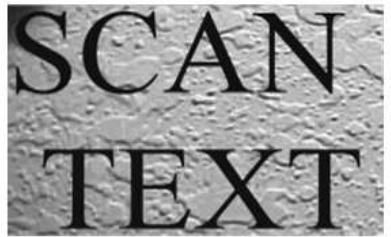

b. $k_{s}=1.3$

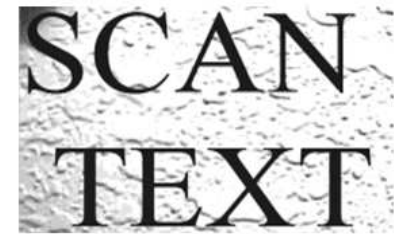

d. $k_{\mathrm{s}}=0.6$

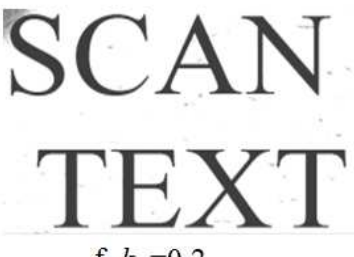

f. $k_{\mathrm{s}}=0.2$
Fig. 4: Different contrast levels at different values of $k_{s}$

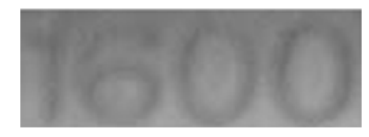

a. Input image.

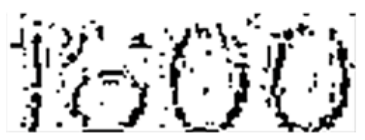

c. OT at $\mathrm{w}=5$

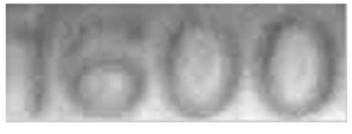

b. Contrast stretched image at $\mathrm{k}_{\mathrm{s}}=0.8$

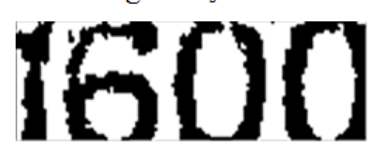

d. OT at $w=15$

Fig. 5: Result of different steps of OT at different window size with $k_{c}=0.03$

$T(x, y)=m_{b}(x, y)\left[1+k_{c}(\partial-1)\right]$

Where:

$m_{b}(x, y)=$ is the BBPM as in (7) $\partial=S(x, y)-m_{b}(x, y)$

$k_{c}=$ is a bias which controls the background noise removal as shown in the Fig. 4

With this threshold value, binarization is proceeded based on the contrast stretched image $S$ as in (8) as:

$B(x, y)=\left\{\begin{array}{cc}1 & \text { if } S(x, y) \geq T(x, y) \\ 0 & \text { otherwise }\end{array}\right.$

where $B$ is the binarized image.

This technique can be summarised as the following algorithm: 


\section{Algorithm}

1. Take the input image $I$

2. Obtain the contrast stretched image $\mathrm{S}$ of $\mathrm{I}$ as in (8).

3. Calculate BBPM $m_{b}(x, y)$ of a local block $w \times w$ as $S(x, y)$ at centre using $(7)$.

4. Calculate threshold value $T(x, y)$ using (9)

5. Transform $S(x, y)$ to its corresponding binarized value $B(x, y)$ using $(10)$

6. Repeat step 3 to 5 until the last pixel of the input image.

As the local BBPM is calculated from the 9 pixels only, its computational time is independent of local block size. Hence it can work as fast as global techniques but result is much better than the global. As compared with other local techniques, it is faster even they use integral sum image for determining local mean as prior process. Not only its speed, it can adapt the low contrast region also with different window size keeping the bias kc at constant as in Fig. 5.

\section{Experimental Result}

The performance of OT is compared with other local techniques regarding computational time as well as binarized result. Table 1 shows the comparison of computational time consumption while Fig. 6 shows the comparison of binarized results. From the experimental result, it is found that this proposed system is faster than the other related techniques even they use integral sum/average image as prior process to calculate local mean.

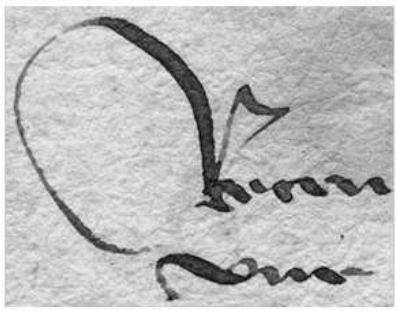

a. Input

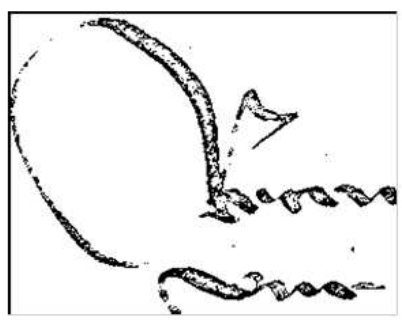

c. TR Singh at $k=0.3$

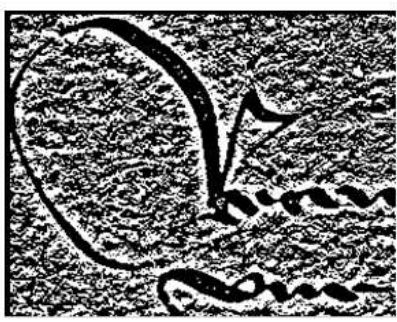

e. Niblack at $\mathrm{k}=0.3$

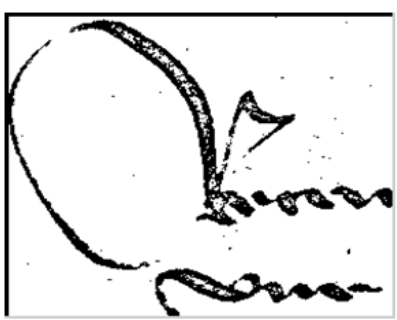

g. Sauvola at $\mathrm{k}=0.3$

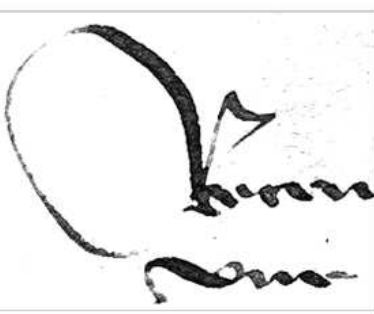

b. Contrast image at $\mathrm{k}_{\mathrm{s}}=0.5$

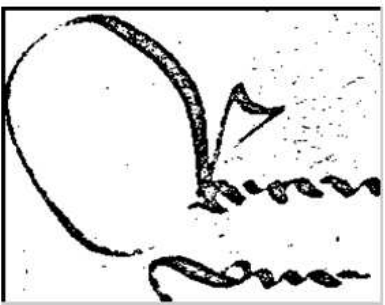

d. $\mathrm{LAAB}$ at $\mathrm{k}=0.54$

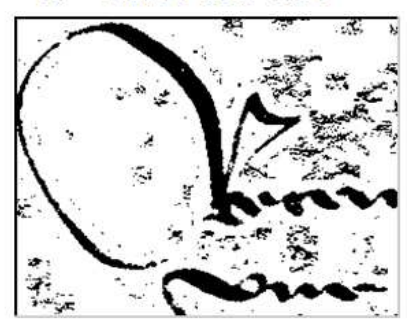

f. Bernsen at $\mathrm{k}=0.3$

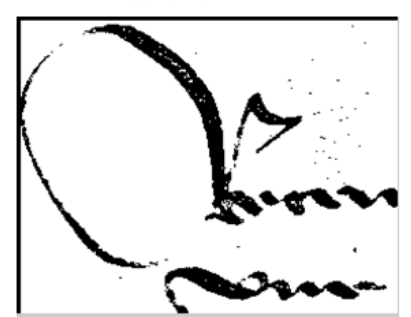

h. OT at $\mathrm{k}_{\mathrm{c}}=0.15$

Fig. 6: Result of different techniques at $w=15$ 
Table 1: Computational Time( in sec) of different technique at different window size $w$

\begin{tabular}{llllllll}
\hline$w$ & OI & Bernsen & LAAB & Niblack & TR & Sauvola & OAT \\
\hline 3 & 0.78 & 0.47 & 3.0 & 5.8 & 2.6 & 5.80 & 0.35 \\
7 & 0.80 & 0.50 & 3.0 & 5.9 & 2.7 & 5.85 & 0.35 \\
15 & 0.82 & 0.56 & 3.1 & 6.0 & 2.8 & 6.00 & 0.35 \\
\hline
\end{tabular}

Not only the computational time, it gives better result as shown in the figures. So as to remove the background noise, other techniques fail to recover low contrast foreground pixel values while OT can recover. The uses of contrast stretching as prior process, it can adapt low contrast region. Since it uses only 9 pixels to determine BBPM at different block size, its computational time is independent from local block size. So its computational time complexity is $O\left(n^{2}\right)$ while other techniques have $O\left(n^{2} \times w^{2}\right)$. If $w=3$, OT has same computational time. From the figures and table, we conclude that this proposed technique is outperformed.

\section{Conclusion}

The proposed technique is a local adaptive binarization technique which can adapt low contrast environment and its computational time is free from local block size. It uses only 9 pixels as neighboring local pixels to adapt the environment without regarding the block size. So as to have more convenient for low contrast region adaptation, contrast stretching is carried out as prior process. Since it uses only 9 pixels, its computational time complexity is fixed at a constant and hence it is free from local block size. As compared with the other related techniques, it outperformed. Since it uses only nine pixels, if the local block is large, there is a problem with the edge region.

\section{Acknowledgement}

We thank to all the authors regarding subject matter for giving us knowledge towards development of this paper in the field of image binarization for publication to journal. We also thank the reviewers of this journal for their suggestion to make stream lining this paper for publication.

\section{Author's Contributions}

O. Imocha Singh: He took overall care of preparation for this paper by drafting and participating in all experiments, co-coordinating the data-analysis. He review it critically for significant intellectual content.

T. Romen Singh: He contributes in drafting the article and technical supports in implementing the various experiments for the paper.

\section{Ethics}

This article is original and contains unpublished material. The corresponding author confirms that all of the other authors have read and approved the manuscript and there are no ethical issues involved.

\section{References}

Bernsen, J., 1986. Dynamic thresholding of gray-level images. Proceedings of the 8th International Conference on Pattern Recognition. Paris, pp: 1251-1255.

Cattoni, R., T. Coianiz, S. Messelodi and C.M. Modena, 1998. Geometric layout analysis techniques for document image understanding. A Review Tech. Rep. IRST, Trento, Italy.

Chi, Z., H. Yan and T. Pham, 1996. Fuzzy algorithms with applications to image processing and pattern recognition. World Scientific Publishing.

Chow, C.K. and T. Kaneko, 1972. Automatic detection of the left ventricle from cineangiograms. Comput. Biomed. Res., 5: 388-410. DOI: $10.1016 / 0010-4809(72) 90070-5$

Eikvil, L., T. Taxt and K. Moen, 1991. A fast adaptive method for binarization of document images. Proc. ICDAR, France, pp: 435-443.

Gorman, L.O., 1994. Binarization and multithresholding of document images using connectivity. CVGIP, Graph Models Image Process, 56: 494-506.

DOI: 10.1006/cgip.1994.1044

Liu, Y. and S.N. Srihari, 1997. Document image binarization based on texture features. IEEE Pattern Anal. Mach. Intell., 19: 540-544. DOI: $10.1109 / 34.589217$

Mardia, K.V. and T.J. Hainsworth, 1988. A spatial thresholding method for image segmentation. IEEE Trans. Pattern Anal. Mach. Intell., 10: 919-927. DOI: $10.1109 / 34.9113$

Niblack, W., 1986. An Introduction to Digital Image Processing. Prentice-Hall, Englewood Cliffs, NJ, pp: 115-116.

Otsu, N., 1979. A threshold selection method from graylevel histograms. Proceedings IEEE Trans. Systems, Man Cybernetics, 9: 62-66. DOI: $10.1109 /$ TSMC.1979.4310076

Sauvola, J. and M. Pietikainen, 2000. Adaptive document image binarization. Pattern Recognition, 33: 225-236. DOI: 10.1016/S0031-3203(99)00055-2

Shafait, F., D. Keysers and T.M. Breuel, 2006. Performance comparison of six algorithms for page segmentation. Proceedings of the 7th IAPR Workshop on Document Analysis Systems, Nelson, New Zealand, pp: 368-379. DOI: $10.1007 / 1166948733$ 
Singh, O.I., T. Sinam, O. James and T.R. Singh, 2012. Local contrast and mean thresholding in image binarization. Int. J. Comput. Appli.

Singh, R.T., S. Roy and K.M. Singh, 2012. Local Adaptive Automatic Binarization (LAAB). Int. J. Comput. Appli.

Singh, R.T., S. Roy, O.I. Singh, T. Sinam and K.M. Singh, 2011. A new local adaptive thresholding technique in Binarization. IJCSI, 8: 271-277.

Taxt, T., P.J. Flynn and A.K. Jain, 1989. Segmentation of document images. IEEE Trans. Pattern Anal. Mach. Intell., 11: 1322-1329.

DOI: $10.1109 / 34.41371$
Viola, P. and M.J. Jones, 2004. Robust real-time face detection. Int. J. Computer Vision, 57: 137-154. DOI: 10.1023/B:VISI.0000013087.49260.fb

White, J.M. and G.D. Rohrer, 1983. Image thresholding for optical character recognition and other applications requiring character image extraction. IBM J. Res. Development, 27: 400-411. DOI: $10.1147 / \mathrm{rd} .274 .0400$

Yanowitz, S.D. and A.M. Bruckstein, 1989. A new method for image segmentation. Comput. Vis. Graph. Image Process., 46: 82-95. DOI: $10.1016 / \mathrm{S} 0734-189 X(89) 80017-9$ 